Chirurg 2019 $\cdot 90: 758$

https://doi.org/10.1007/s00104-019-0997-y

Online publiziert: 25. Juni 2019

(c) Springer Medizin Verlag GmbH, ein Teil von Springer Nature 2019

\section{Originalpublikation}

Amelung FJ, Borstlap WAA, Consten ECJ et al (2019) Propensity score-matched analysis of oncological outcome between stent as bridge to surgery and emergency resection in patients with malignant left-sided colonic obstruction. BJS https://doi.org/10.1002/bjs. 11172

Hintergrund. Malignome sind die häufigste Ursache des akuten Kolonverschluss. In dieser Situation erfolgt häufig die notfallmäßige, offene Resektion oft einhergehend mit einem erheblichen Verlust an Kolonstrecke und der Anlage eines Stomas. Eine Alternative in besteht in der endoskopischen Platzierung eines selbstexpandierenden Metallstents (SEMS) zur Dekomprimierung und Tonisierung des Darms, um die onkologische Resektion unter günstigeren Bedingungen durchzuführen („bridge to surgery“, BTS). Aufgrund einer möglicherweise erhöhten Rezidivrate ist diese Option für Patienten, deren Allgemeinzustand eine Operation zulässt, bislang in keiner Leitlinie empfohlen.

Methoden. Für diese Studie wurden Daten von Patienten mit maligner linksseitiger Kolonobstruktion aus den Jahren 2009 bis 2016 ausgewertet. Da keine Randomisierung erfolgte, wurde versucht, den Einfluss von Störfaktoren mittels 1:2 „propensitiy score matching“ $\mathrm{zu}$ reduzieren. Der primäre Endpunkt bestand im Gesamtüberleben (OS) und im krankheitsfreien Überleben (DFS) über einen Zeitraum von 3 Jahren.

M. Schrempf $\cdot$ M. Anthuber

Klinik für Allgemein-, Viszeral-Transplantationschirurgie, Klinikum Augsburg, Augsburg, Deutschland

\title{
Onkologisches Outcome nach Notfallresektion vs. Stenting bei maligner linksseitiger Kolonobstruktion
}

Ergebnisse. Nach „propensity score matching“ wurden 222 Patienten mit SEMS und 444 Patienten mit notfallmäBiger Operation („emergency resection“, ER) ausgewertet und verglichen. Die Perforationsrate in der SEMS-Gruppe betrug $7,7 \%$ (17 von 222). Die Lokalrezidivrate unterschied sich nicht zwischen der SEMS- und ER-Gruppe (11,4\% vs. $13,6 \% ; p=0,45)$. Das 3-Jahres-DFS (SEMS 58,8\% vs. ER 52,6\%; $p=0,18$ ) und das 3-Jahres-OS (SEMS $74 \%$ vs. ER $68,3 \% ; p=0,23$ ) unterschied sich ebenfalls nicht zwischen den beiden Gruppen. Die Therapie mit SEMS als BTS resultierte in einer signifikant niedrigeren Rate an permanenten Stomaanlagen $(23,9 \%$ vs. $45,3 \%$; $p<0,001)$. Dieser Effekt trat bei Patienten über 70 Jahre verstärkt in Erscheinung (29\% vs. $57,9 \% ; p<0,0001)$. Im Falle einer Perforation betrug die Lokalrezidivrate für Patienten in der SEMS-Gruppe $18 \%$ gegenüber $11 \%$ in Gruppe ohne Perforation $(p=0,43)$. Das 3-Jahres-OS betrug in der SEMS-Gruppe mit Perforation $61 \%$ und ohne Perforation $75,1 \%$ $(p=0,53)$.

Diskussion und Fazit. In dieser Arbeit zeigte sich kein Unterschied im onkologischen Outcome zwischen der SEMSund der ER-Gruppe. In mehreren Arbeiten war die Platzierung eines SEMS mit einer erhöhten Rezidivrate und einem schlechteren OS assoziiert. Demgegenüber stehen die Ergebnisse zweier Metaanalysen, die keine Unterschiede im OS feststellen konnten [1, 2]. Der Vor- teil des Stentings besteht in der Reduktion permanenter Stomaanlagen und in der Möglichkeit, nach Entlastung des Kolons den Eingrifflaparoskopisch mit allen Vorteilen der minimal-invasiven Chirurgie durchzuführen. Die Ergebnisse dieser Studie zeigen, dass die Stenteinlage als BTS eine Alternative zur Notfallresektion darstellen kann. Voraussetzung hierfür ist jedoch eine spezialisierte Endoskopieabteilung. Komorbiditäten des Patienten und der Patientenwunsch nach einer ausführlichen Aufklärung über die Vor- und Nachteilebeider Methoden sind bei der Verfahrenswahl unbedingt zu berücksichtigen.

\section{Korrespondenzadresse}

Prof. Dr. M. Anthuber

Klinik für Allgemein-, Viszeral-Transplantationschirurgie, Klinikum Augsburg Stenglinstr. 2, 86156 Augsburg, Deutschland matthias.anthuber@klinikum-augsburg.de

Interessenkonflikt. M. Schrempf und M. Anthuber geben an, dass kein Interessenkonflikt besteht.

\section{Literatur}

1. Amelung FJ, Burghgraef TA, Tanis PJ et al (2018) Critical appraisal of oncological safety of stent as bridge to surgery in left-sided obstructing colon cancer; a systematic review and meta-analysis. Crit Rev Oncol Hematol 131:66-75. https://doi.org/10. 1016/j.critrevonc.2018.08.003

2. Ceresoli M, Allievi N, Coccolini F et al (2017) Longterm oncologic outcomes of stent as a bridge to surgery versus emergency surgery in malignant left side colonic obstructions: a meta-analysis. J Gastrointest Oncol 8:867-876. https://doi.org/ 10.21037/jgo.2017.09.04 